\title{
Language in the genes: Where's the evidence?
}

\author{
Ocke-Schwen Bohn \\ Aarhus University
}

\begin{abstract}
Evidence regarding the genetic bases of human language abilities comes from many sources, but none is as rich and reliable as the one that comes from infant speech perception studies. This contribution provides an overview of how research on infant speech perception informs the debate on the genetic basis of human language abilities. Specifically, this contribution reviews findings which document infants' abilities to learn from pre- and postnatal experience, and findings which strongly suggest that humans possess language-specific abilities as part of their genetic makeup.
\end{abstract}

\section{Introduction}

Linguists disagree on many things, but there is perhaps no divide as deep as the one between those who view linguistic knowledge as largely shaped by experience with the ambient language(s) and learned through the application of general cognitive principles, and those who claim that the linguistic knowledge of humans is genetically based. Traditionally, the evidence that the "geneticists" bring to bear on this issue comes from (putative) language universals, from the assumption that the learning of native languages (L1s) is fast, and from the claim that negative feedback does not play any role in L1 acquisition (Vikner 2001).

The problem with these three sources of evidence is that they are not as solid as the genetic camp would like them to be. Language universals can be specifically linguistic, but in many cases the jury is still out on whether alternative accounts, such as those that invoke a cognitive or functional basis for language universals, are not more valid (Haspelmath 2008). The claim that L1 acquisition is fast depends very much on what is meant by "fast"; the evidence clearly shows that children in their second decade

Ken Ramshøj Christensen, Henrik Jørgensen \& Johanna L. Wood (eds.). 2019.

The Sign of the V-Papers in Honour of Sten Vikner.

Dept. of English, School of Communication \& Culture, Aarhus University, pp. 77-93, doi:10.7146/aul.348.90. (C) The author(s) 
of life do not yet have the same linguistic knowledge as adults do (C. Chomsky 1969; Hazan \& Barrett 2000). A recent large-scale study even reported that "native speakers did not reach asymptote until around 30 years old" (Hartshorne, Tenenbaum \& Pinker 2018: 269). This is certainly fast in geologic terms, but slow in terms of human development. Finally, the claim that L1 learning takes place in the absence of negative evidence (which learners receive when they produce ungrammatical utterances) is one of the most hotly debated issues in L1 acquisition research. While both parents and psycholinguists know that attempts to provide children with explicit direct negative evidence (in which the learner is explicitly told what is wrong) are wasted on the learner, L1 acquisition researchers agree that the input of child learners contains implicit direct negative evidence (in which the learner is exposed to an adult reformulation of her utterance). The unresolved issue of contention is whether L1 learners (can) use this type of negative evidence to learn their language (Saxton 2000).

A sympathetic evaluation of the traditional arguments for a genetic basis of linguistic abilities would have to conclude that the three pillars on which they rest (universals which do not have a general cognitive/functional basis, "fast" language acquisition, irrelevance of negative feedback) lack a solid empirical foundation. Still, the facts that humans are the only species that communicates through language and that language acquisition is highly regular and (near-)universal in our species makes it seem logical to assume that the species-specific trait "language" must have a genetic basis. But where is the evidence for this? An obvious area of research to consider in the pursuit of this question are studies of infants' (pre-) linguistic abilities. Infants' linguistically relevant abilities are due either to early exposure to the ambient language(s), or to the fact that evolution has prepared human infants to acquire any language. Much research on infant speech perception has been motivated by a strong interest in teasing apart the effects of early experience on the one hand and innate abilities on the other. This contribution provides an overview of how research on infant speech perception informs the debate on the genetic basis of human language abilities.

The structure of this chapter mirrors the chronology of speech perception development over the first year, with an outlook on later speech perception abilities in adolescence and adulthood. Section 2 provides an overview of linguistically relevant abilities at or around birth, and section 3 examines how early experience interacts with innate abilities over the first year of life and beyond. 


\section{Linguistically relevant abilities at (or around) age 0}

Two reasons can be given for the somewhat unprecise title of this section, in which "around" covers late prenatal and early postnatal ontogenesis: First, the fetal auditory system is functional during the final prenatal trimester (Lickliter 1993), which makes it necessary to include the prenatal period in any discussion of infants' linguistically relevant abilities. Second, the "around" reflects the tradition in the infant literature to refer to infants up to the age of 8 weeks as "newborn", which is justified because of the very different behavioral, cognitive, and neuropsychological characteristics of newborns, thus defined, from infants two months and older (Watson, Robbins \& Best 2014).

The abilities which newborns demonstrate are usually interpreted as being due to either prenatal experience or genetic endowment, with the important qualification that external stimulation can only become experience if the stimulated organism is genetically predisposed to turn stimulation into experience. So, which linguistically relevant abilities and biases do newborns possess?

\subsection{Global properties of speech}

Newborns enter this world with broad predispositions and with experiencebased knowledge which both indicate that some of the prerequisites for language learning are in place already at birth. Newborns discriminate speech from nonspeech (Alegria \& Noirot 1982), and they prefer to listen to normal speech as opposed to speech played backwards, filtered speech, or sine-wave analogues of speech (Dehaene-Lambertz, Dehaene \& HertzPannier 2002; Vouloumanos \& Werker 2007). However, they broadly prefer to listen to primate vocalizations and only later, at three months of age, narrow their preferences down to human speech (Voloumanos et al. 2010). With respect to more specific biases, it has been known for some time that newborns prefer to listen to their mother's voice (Mehler et al. 1978). More recently, Voegtline et al. (2013) measured the response (heart rate, movement in utero) in fetuses at 36 weeks gestation and found that the fetuses demonstrated maternal voice recognition. The attentiveness of fetuses to the nonsegmental properties of speech to which they have access in the low-pass filter environment of the womb, i.e., rhythm and intonation, is further evidenced by the preference of newborns to listen to infant directed as opposed to adult directed speech (Cooper \& Aslin 1990), most likely because in many cultures, infant directed speech is characterized by higher and more varied pitch (Fernald et al. 1989; but see Bohn 2013). The prenatal attentiveness to pitch changes was also demonstrated in study 
by Partanen et al. (2013), who reported that newborns display mismatch responses in response to pitch changes in speech-like auditory stimuli heard before birth. The sensitivity of fetuses to rhythmic properties of speech is evidenced by newborns' preference for their mother's language (if the mother is monolingual) or languages (if the mother is bilingual) as shown by Byers-Heinlein, Burns \& Werker (2010) for rhythmically distinct languages such as English and Tagalog. Interestingly, a study by Moon, Lagercrantz \& Kuhl (2012) revealed that the language experience of fetuses is not restricted to nonsegmental properties because infants born to American English women or to Swedish-speaking women responded differently (sucking rate) to vowels from the native as opposed to the nonnative language.

Except for the very broad and apparently genetically based preference for sounds produced by human(-like) vocal tracts, the above brief overview suggests that the newborn's linguistically relevant preferences are all based on prenatal experience with the mother's speech characteristics. Clearly, these prenatally shaped preferences prepare and aid the infant in her species-specific task of acquiring the ambient language(s). However, the prenatal stimulation could not become experienced-based linguistically relevant knowledge if the infant did not have the ability to process these stimuli. Part of the genetic basis of this ability has been well documented for a long time. For instance, Molfese (1977) reported that newborns show cerebral specialization for speech (left hemisphere) and nonspeech (right hemisphere). A more recent study localized speech processing in the newborn more narrowly and found, using fMRI, a left-lateralized response in the temporal cortex for speech compared to biological non-speech sounds, indicating that this region is selective for speech by the first month of life (Shultz et al. 2014). This and similar findings for 3-month-olds (Homae, Watanabe \& Taga 2014) is not only informative regarding the locus of speech processing very early in life. It also supports the "speech is special" claim of the Motor Theory of speech perception, which postulates that the processes by which humans decode linguistic messages from the acoustic signal are different from auditory processes used to perceive non-speech acoustic signals (Liberman et al. 1967). For adults, there is convincing behavioral and neurological evidence that the human perceptual system responds differently to speech as opposed to general auditory input (e.g., Mattingly et al. 1971; Van Lancker \& Fromkin 1973; Best \& Avery 1999), and the studies just cited strongly suggest that this specialization for speech is part of our genetic makeup. 


\subsection{Specific properties of speech}

Perhaps one of the most solid findings from research on early infant development concerns the ability of infants to discriminate stimuli from consonant continua in a categorical fashion (Eimas et al. 1971), no matter whether the contrast is used in the infant's ambient language(s) (Lasky, Syrdal-Lasky \& Klein 1975; Streeter 1976). What is meant by "categorical fashion" is that infants, just like adults, do not discriminate just any two acoustically distinct stimuli, rather, they discriminate just those stimuli which straddle the boundary between two categories as established in adult perception experiments. Until recently, this finding has been replicated for just about any consonant contrast on which infants have been tested, and it has been found in infants right after they were born (for a review, see Eimas 1985), which very strongly suggests that the infant ability to discriminate consonant contrasts categorically is part of their genetic makeup.

The importance of this finding for developmental psychology and psycholinguistics was and still is enormous, because it radically changed the view of infants' abilities that was prevalent in the first half of the last century and beyond. This view was expressed by Fry (1966: 198) as "the child begins by being insensible to differences among speech sounds ... a vital part of language-learning in the early stages is the process by which he becomes sensitive to more and more differences among sounds". Clearly, this empiricist view, for which empirical evidence did not exist at the time of Fry's claim, is wrong. However, the very well documented fact that, to re-write Fry, the child begins by being sensible to differences among speech sounds has to be qualified for the present discussion of infants' innate linguistically relevant abilities.

The first qualification has to acknowledge comparative studies which have shown that some of the contrasts which newborns discriminate categorically are also categorically discriminated by other animals. For example, Kuhl \& Miller (1975) showed that chinchillas, whose peripheral auditory system is quite similar to that of humans, equivalence-classified stimuli from a voice onset time continuum in much the same way as human adults, with a steep labeling function and a boundary located very near the boundary of what humans classify as [da] vs. [ta]. This suggests that, at least with respect to the syllable-initial voicing contrast for stop consonants, human infants exploit general capacities of the mammalian auditory system (see also Kuhl 1981).

The second qualification considers what, at first sight, could be viewed as a partial rehabilitation of Fry's (1966) global claim. Several recent 
studies seem to suggest that it is not the case that infants can discriminate all consonant contrasts in a categorical fashion, no matter whether the contrast is used in the infant's ambient language(s). For example, Narayan, Werker \& Beddor (2010) found that English-learning infants could not discriminate the syllable-initial [na-na] contrast at any of the tested ages up to 12 months, but Filipino-learning infants could, though first at the age of 10-12 months (not at 6-8 months). Because Filipino, but not English, has this contrast, Narayan, Werker \& Beddor interpreted this finding as suggesting that acoustic salience (which is low for the [na-na] contrast) affects the ability of infants to discriminate consonant contrasts, and that language experience facilitates discrimination of acoustically similar distinctions. This interpretation is further supported by Sato, Sogabe \& Mazuka (2010), who reported that Japanese-learning infants do not discriminate vowel length contrasts (which are phonemic in Japanese) until the age of ca. 8 months. Likewise, Sato, Kato \& Mazuka (2012) found that Japanese-learning infants acquire sensitivity to contrasts of single/ geminate obstruents first by 9.5 months of age. Further support for the view that the ability to discriminate contrasts which are not particularly salient needs to be learned and is not innate, comes from a study by Polka, Colantonio \& Sundara (2001), who reported that English-leaning infants' discrimination of [d]-[ð] is poor, and from a study by Shin, Choi \& Mazuka (2018), who found that Korean-learning infants do not discriminate the Korean plain-tense [s-s*] contrast until the age of 7-9 months.

However, a recent study casts doubt on the revisionist view that infants' ability to discriminate contrasts is restricted to acoustically salient contrasts, and that subtle contrasts depend on language experience. Sundara et al. (2018) attempted to replicate the findings of Narayan, Werker \& Beddor (2010). In one experiment, Sundara et al. used the stimuli employed by Narayan, Werker \& Beddor in a very similar procedure which, however, differed in that it was fully infant-controlled. Sundara et al. (2018) reported that, using this more sensitive paradigm, English-learning children could indeed discriminate the syllable-initial [na-na] contrast at 4 months of age, unlike what Narayan, Werker \& Beddor (2010) had reported using a less sensitive non-infant controlled paradigm. Additionally, Sundara et al. (2018) showed that both French-learning and English-learning infants could discriminate the acoustically not very salient Tamil dental-retroflex contrasts for both nasals and laterals at 6 months of age. Even though the infants in the Sundara et al. study were not newborns, these findings show that early experience is not necessary for the ability to discriminate 
subtle consonant contrasts. This suggests that, to conclude, the evidence contradicts Fry (1966): The child does indeed begin by being sensible to differences among speech sounds.

This section has focused on consonant perception because the research on vowel perception in newborns and older infants has primarily addressed sets of questions that relate to the perceptual narrowing pattern (see section 3.1 ), the characteristics, origins, and functions of perceptual asymmetries (see section 3.2), and bimodal/intermodal speech perception in infancy. The latter question was first addressed by Kuhl \& Meltzoff (1982; 1984) who examined at what age infants, like adults, are intermodal perceivers who exploit and integrate information about speech from the auditory and the visual channel. The finding by Kuhl \& Meltzoff $(1982 ; 1984)$ that 5 months old infants recognize the correspondence between auditorily and visually presented speech sounds (for the extreme vowels [i] and [a]) pointed to an early link between the channels and between the production and perception of vowels. At first sight, it could be argued that this link is not specific to speech sounds because infants at that age also successfully integrate visual and auditory information for the perception of nonspeech events such as a sound burst and a visual impact (Spelke 1979, see also Bahrick 1983). However, more recent studies have pushed the age at which this link can be observed further down to 4 months (Bahrick, Netto \& HernandezKeif et al. 1998; Patterson \& Werker 2002). The finding by Patterson \& Werker (2003) that infants as young as 2 months provide robust evidence of matching vowel information in face and voice was interpreted by the authors as supporting arguments for "some kind of privileged processing or particularly rapid learning of phonetic information". The privileged processing would point to a genetic origin of this ability, and even the rapid learning would suggest that the speed at which this learning takes place is possible only if it builds on some kind of predisposition.

\section{Infant speech perception from newborn to toddler (and beyond) 3.1 Perceptual narrowing}

Much of the research on infant speech perception after the newborn stage has focused on the question of when infants, who initially are universal perceivers, become language-specific listeners, and, more specifically, the chronology of different aspects of speech perception changes (e.g., for different consonant classes, for vowels, for prosodic properties). At first sight it could appear that the infant age range between newborn (up to ca. 2 months) and toddler (ca. 12 months) has little to offer for any discussion of the genetic basis of linguistically relevant knowledge because this age 
is largely characterized by a perceptual narrowing pattern during which infants tune in to native speech properties. This attunement first affects prosodic properties: Whereas newborns can only discriminate languages from different rhythmical classes like English and French, 5-month olds discriminate languages from the same rhythmical class, e.g., English and Dutch (Nazzi \& Ramus 2003; Nazzi, Jusczyk \& Johnson 2000). Between 6 and 12 months, infants become worse at discriminating consonant contrasts which do not occur in their native language (for a review, see Werker \& Tees 2005, for an interesting exception see Best, McRoberts \& Sithole 1988) and they show improved discrimination of native contrasts (e.g., Kuhl et al. 2006), indicating perceptual elaboration as a function of experience with the native language(s). The findings for vowels are less clear: Withincategory discrimination is clearly affected by the ambient language at 6 months of age (Kuhl et al. 1992) and a meta-analysis by Tsuji \& Cristia (2014) revealed a similar (but earlier) perceptual narrowing pattern for vowels as for consonants. However, this pattern is not confirmed by all studies: Polka \& Bohn (1996) found that cross-category discrimination of native and nonnative vowels did not change for English- and for Germanlearning infants between the ages of 6 and 12 months.

Overall, the speech perception development between the newborn and the toddler age is characterized by a maintenance of discrimination abilities for those sounds that occur contrastively in the ambient language(s), and a "loss" of abilities that do not. The quotation marks around "loss" are important and highly relevant to the topic of this contribution, because Werker (1989), who originally characterized the role of experience as leading to either maintenance or "loss" of perceptual abilities, later made it clear that "developmental change does not involve loss" (Werker 1994: 93). This is an important point because what happens in the second half of the first year of life is a reversible shift of attention away from those acoustic cues that are not phonologically informative. There is a very large body of research, especially on cross-language and second language speech perception, which clearly shows that the universal perceptual abilities that all humans had as newborns are never completely lost (e.g., due to neurophysiological ageing), but remain latent and can be re-learned, through immersion or perceptual training, at any of the adult ages which have been examined (for a review, see Bohn 2018). A more appropriate characterization of the influence of the ambient language on speech perception in the second half of the first year of life (instead of maintenance vs. "loss" of initial, most likely innate abilities) would be maintenance 
vs. latency. This characterization is not just supported by studies of L2 speech perception but also by studies which examined the relearning of perceptual abilities in international adoptees who were exposed to a native sound system in infancy, then grew up in a language environment with no exposure to native sound contrasts, and still showed native-like perception after many years of zero exposure (e.g., Au et al. 2002; Oh, Au \& Jun 2010; Choi, Cutler \& Broersma 2017).

\subsection{Maintenance of perceptual biases}

Another phenomenon which points to a species-specific and thus perhaps genetically based aspect of human language learning ability was first described by Polka \& Bohn(1996), who observed that both English-learning and German-learning infants are biased vowel perceivers. As confirmed by a series of later studies (e.g., Bohn \& Polka 2001; Polka \& Bohn 2003; 2011), vowels which are peripheral in the universal human articulatory/ acoustic vowel space have a special status vis-à-vis less peripheral vowels, e.g., the more peripheral English [æ] as opposed to the less peripheral English $[\varepsilon]$ vowel. As is customary in many infant speech perception studies, we used a change/no change paradigm, in which both Englishlearning, German-learning, and Danish-learning children consistently were much better at discriminating a vowel contrast if the change was presented from a less peripheral to a more peripheral vowel (e.g., [y] to $[\mathrm{u}]$ ) than from a more peripheral to a less peripheral vowel (e.g., $[\mathrm{u}]$ to [y]). A review of the literature revealed that this perceptual asymmetry favoring relatively peripheral vowels was observed (but not interpreted) in several other studies with different methodologies (regarding procedures, types stimuli, participants), which led us to propose the Natural Referent Vowel (NRV) framework (Polka \& Bohn 2011). Research inspired by this framework addresses a range of questions including those regarding the origin, the species-specificity, and the maintenance or loss of this bias beyond infancy.

Two of the questions addressed within the NRV framework are highly relevant in the context of the topic of this contribution, namely, species-specificity and maintenance or loss of the perceptual bias favoring relatively peripheral vowels in infant speech perception. Regarding the question of whether the perceptual biases observed with human infants are unique to our species, the review of the relevant literature by Polka $\&$ Bohn (2003) revealed that the perceptual asymmetries which had been observed in non-human species (cats and blackbirds, see Hienz, Sachs \& Sinnott 
1981 and Hienz, Alesczyk \& May 1996) are a function of just one acoustic parameter of the stimuli, namely, the frequency of the second formant (F2), which is very different in nature from asymmetries observed in infant speech perception studies: For infants, vowels that serve as attractors in perceptual asymmetries are those which are relatively more peripheral in the human vowel space. This is acoustically much more complex than the simple change in $\mathrm{F} 2$ because it can be signaled by an increase or a decrease in either F1 and/or F2. Polka \& Bohn $(2003 ;$ 2011) suggest that this difference between human infants and non-human animals can be taken as indication of a special adaptation to the human vowel space in humans. It should be noted however, that these perceptual biases have not yet been tested in infants younger than 4 months, so the alternative interpretation that the special status of relatively peripheral vowels could be experiencedbased (through infant-directed speech, see Kuhl et al. 1997, or by exposure to typical facial expressions mothers direct to their infants, which are the visual equivalents of corner vowels, see Chong et al. 2003), cannot be ruled out.

Regarding the maintenance or loss of the perceptual bias favoring relatively peripheral vowels in infant speech perception, a hypothesis developed using the NRV framework is that these biases will be lost if nonfunctional because the ambient language(s) provide(s) experience with both members of the contrast, but will be maintained if the ambient language(s) do not provide this experience. This hypothesis has been confirmed, for instance in studies of the discrimination of the [u-y] vowel contrast, which English-learning and German-learning infants discriminate asymmetrically. English-speaking adults, who are not exposed to this contrast, maintain this asymmetry, whereas German adults, in whose language this contrast is phonemic, do not show this asymmetry. These and other results summarized in Polka \& Bohn (2011; see also Bohn \& Polka 2014; Polka, Bohn \& Weiss 2015), show how innate propensities and native language experience may interact.

The infant vowel perception research briefly referred to above suggests, to paraphrase Nam \& Polka (2016: 57), that "the phonetic landscape in infant ... perception is an uneven terrain". A recent meta-analysis by Tsuji \& Cristia (2017) has solidly confirmed the basic tenet of the NRV framework, namely, that infants are not blank slates as far as vowel perception is concerned. But what about consonants? Are there consonants which have a special status in both infant and adult speech perception and which thus suggest innate predispositions? The question of whether natural referent 
consonants exist has only recently been explored in just a few infant and adult perception studies. In general, and to the extent that generalization is possible, the evidence so far suggests that the alveolar place of articulation has this special status, no matter whether the manner of articulation is stop, fricative, affricate, or approximant. (For infants, see Tsuji et al. 2015; for adults, see Cutler, Weber \& Otake 2006; Lai 2009; Bundgaard-Nielsen et al. 2015; Schluter, Politzer-Ahles \& Almeida 2016.) Overall, these studies suggest that alveolars are somehow "better" consonants for both L1 and L2 learners. More research is clearly needed, but the findings reported so far carry the promise of providing a psycholinguistic basis for descriptive notions such as "underspecification" and "markedness".

\section{Conclusion}

The aim of this contribution was to review infant speech perception research for evidence addressing the question of a genetic basis of linguistically relevant abilities. This review showed that newborns have already prenatally learned about the global properties of the ambient language(s). They seem to be biologically well prepared to process linguistically relevant information because the left temporal cortex in the fetal human brain is specialized to process speech as opposed to nonspeech sounds. At the earliest possible age that infants can be tested, they demonstrate an innate ability to discriminate consonant contrasts, no matter whether these contrasts occur in the ambient language(s) or not. This ability is never lost, it remains latent and can be re-acquired at any age. For infants, the phonetic landscape is uneven, with certain speech sounds having a universally privileged status. This apparently innately skewed perception of speech sounds can also be observed in adults. In conclusion, research on speech perception provides clear evidence that humans are not blank slates. An important part of our species-specific ability to learn and use language is indeed in the genes.

\section{References}

Alegria, Jesus \& Eliane Noirot. 1982. Oriented mouthing activity in neonates: Early development of differences related to feeding experiences. In Jacques Mehler, Jacques, Edward Walker \& Merrill Garret (eds.), Perspectives on mental representation, 389-397. Hillsdale, NJ: Erlbaum.

Au, Terry K.-F., Leah M. Knightly, Sun-Ah Jun \& Janet S. Oh. 2002. Overhearing a language during childhood. Psychological Science 13(3). 238-243. doi:10.1111/1467-9280.00444. 
Bahrick, Lorraine E. 1983. Infants' perception of substance and temporal synchrony in multimodal events. Infant Behavior and Development 6(4). 429451. doi:10.1016/S0163-6383(83)90241-2.

Bahrick, Lorraine E., Dianelys Netto \& Maria Hernandez-Keif. 1998. Intermodal perception of adult and child faces and voices by infants. Child Development 69(5). 1263-1275. doi:10.2307/1132264.

Best, Catherine T. \& Robert A. Avery. 1999. Left-hemisphere advantage for click consonants is determined by linguistic significance and experience. Psychological Science 10(1). 65-70. doi:10.1111/1467-9280.00108.

Best, Catherine T., Gerald W. McRoberts \& Nomathemba M. Sithole. 1988. Examination of perceptual reorganization for nonnative speech contrasts: Zulu click discrimination by English-speaking adults and infants. Journal of Experimental Psychology: Human Perception and Performance 14(3). 345360. doi:10.1037/0096-1523.14.3.345.

Bohn, Ocke-Schwen. 2013. Acoustic characteristics of Danish infant directed speech. Proceedings of Meetings on Acoustics 19, 060055. doi:10.1121/1.4798488.

Bohn, Ocke-Schwen. 2018. Cross-language and second language speech perception. In Eva M. Fernandez \& Helen S. Cairns (eds.), The handbook of psycholinguistics, 213-239. Hoboken, NJ: John Wiley \& Sons. doi:10.1002/9781118829516.ch10.

Bohn, Ocke-Schwen \& Linda Polka. 2001. Target spectral, dynamic spectral, and duration cues in infant perception of German vowels. Journal of the Acoustical Society of America 110(1). 504-515. doi:10.1121/1.1380415.

Bohn, Ocke-Schwen \& Linda Polka. 2014. Fast phonetic learning in very young infants: what it shows, and what it doesn't show. Frontiers in Psychology 5. 511. doi:10.3389/fpsyg.2014.00511.

Byers-Heinlein, Krista, Tracey C. Burns \& Janet F. Werker. 2010. The roots of bilingualism in newborns. Psychological Science 21(3). 343-348. doi:10.1177/0956797609360758.

Choi, Jiyoun, Anne Cutler \& Miriam Broersma. 2017. Early development of abstract language knowledge: Evidence from perception-production transfer of birth-language memory. Royal Society Open Science 4(1), 160660. doi:10.1098/ rsos. 160660 .

Chomsky, Carol. 1969. The acquisition of syntax in children from 5 to 10. Cambridge, MA: MIT Press.

Chong, S. C. F., Janet F. Werker, James A. Russell, \& James M. Carroll. 2003. Three facial expressions mothers direct to their infants. Infant and Child Development 12. 211-232. doi:10.1002/icd.286.

Cooper, Robin P. \& Richard N. Aslin. 1990. Preference for infant-directed speech in the first month after birth. Child Development 61(5). 1584-1595. doi:10.2307/1130766. 
Dehaene-Lambertz, Ghislaine, Stanislas Dehaene \& Lucie Hertz-Pannier. 2002. Functional neuroimaging of speech perception in infants. Science 298. 20132015. doi:10.1126/science. 1077066.

Eimas, Peter D. 1985. The perception of speech in early infancy. Scientific American 252(1). 46-53. doi:10.1038/scientificamerican0185-46.

Fernald, Anne, Traute Taeschner, Judy Dunn, Mechthild Papousek, Benedicte de Boysson-Bardies \& Ikuko Fukui. 1989. A cross-language study of prosodic modifications in mothers' and fathers' speech to preverbal infants. Journal of Child Language 16(3). 477-501. doi:10.1017/S0305000900010679.

Fry, D. B. 1966. The development of the phonological system in the normal and deaf child. In Frank Smith \& George A. Miller (eds.), The genesis of language, 187-206. Cambridge, MA: MIT Press.

Hartshorne, Joshua K., Joshua B. Tenenbaum \& Steven Pinker. 2018. A critical period for second language acquisition: Evidence from 2/3 million English speakers. Cognition 177. 263-277. doi:10.1016/j.cognition.2018.04.007.

Haspelmath, Martin. 2008. Parametric versus functional explanations of syntactic universals. In Theresa Biberauer (ed.). The limits of syntactic variation. 75-107 Amsterdam: Benjamins. doi:10.1075/la.132.04has.

Hazan, Valerie \& Sarah Barrett. 2000. The development of phonemic categorization in children aged 6-12. Journal of Phonetics 28(4). 377-396. doi:10.1006/ jpho.2000.0121.

Homae, Fumitaka, Hama Watanabe \& Gentaro Taga. 2014. The neural substrates of infant speech perception. Language Learning 64(s2). 6-26. doi:10.1111/ lang.12076.

Jusczyk, Peter W. \& Josiane Bertoncini. 1988. Viewing the development of speech perception as an innately guided learning process. Language and Speech 31(3). 217-238. doi:10.1177/002383098803100301.

Kuhl, Patricia K. 1981. Discrimination of speech by nonhuman animals: Basic auditory sensitivities conducive to the perception of speech-sound categories. Journal of the Acoustical Society of America 70(2). 340-349. doi:10.1121/1.386782.

Kuhl, Patricia K., Jean E. Andruski, Inna A. Chistovich, Ludmilla A. Chistovich, Elena V. Kozhevnikova, Viktoria L. Ryskina, Elvira I. Stolyarova, Ulla Sundberg \& Francisco Lacerda. 1997. Cross-language analysis of phonetic units in language addressed to infants. Science 277. 684-686. doi:10.1126/ science.277.5326.684.

Kuhl, Patricia K. \& Andrew N. Meltzoff. 1982. The bimodal perception of speech in infancy. Science 218. 1138-1141. doi:10.1126/science.7146899.

Kuhl, Patricia K. \& Andrew N. Meltzoff. 1984. The intermodal representation of speech in infants. Infant Behavior and Development 7(3). 361-381. doi:10.1016/ S0163-6383(84)80050-8. 
Kuhl, Patricia K., \& James D. Miller. 1975. Speech perception by the chinchilla: Voiced-voiceless distinction in alveolar plosive consonants. Science 190. 6972. doi:10.1126/science.1166301.

Kuhl, Patricia K., Erica Stevens, Akiko Hayashi, Toshiosada Deguchi, Shigero Kiritani \& Paul Iverson. 2006. Infants show a facilitation effect for native language phonetic perception between 6 and 12 months. Developmental Science 9(2). F13-F21. doi:10.1111/j.1467-7687.2006.00468.x.

Kuhl, Patricia K., Karen A. Williams, Francisco Lacerda, Kenneth N Stevens \& Björn Lindblom. 1992. Linguistic experience alters phonetic perception in infants by 6 months of age. Science 255. 606-608. doi:10.1126/science. 1736364 .

Lasky, Robert E., Ann Syrdal-Lasky \& Robert E. Klein. 1975. VOT discrimination by four to six and a half month old infants from Spanish environments. Journal of Experimental Child Psychology 20(2). 215-225. doi:10.1016/00220965(75)90099-5.

Lickliter, Robert. 1993. Timing and the development of perinatal perceptual organization. In Gerald Turkewitz \& Darlynne A. Devenny (eds.), Developmental time and timing, 105-123. Hillsdale, NJ: Lawrence Erlbaum.

Liberman, Alvin M., Franklin S. Cooper, Donald P. Shankweiler \& Michael Studdert-Kennedy. 1967. Perception of the speech code. Psychological Review 74(6). 431-461. doi:10.1037/h0020279.

Mattingly, Ignatius G., Alvin M. Liberman, Ann K. Syrdal \& Terry Halwes. 1971. Discrimination in speech and nonspeech modes. Cognitive Psychology 2(2). 131-157. doi:10.1016/0010-0285(71)90006-5.

Mehler, Jacques, Josiane Bertoncini, Michele Barriere \& Dora JassikGerschenfeld. 1978. Infant recognition of mother's voice. Perception 7(5). 491-497. doi:10.1068/p070491.

Molfese, Dennis L. 1977. Infant cerebral asymmetry. In Sidney J. Segalowitz \& Frederic A. Gruber (eds.), Language development and neurological theory, 2135. London: Academic Press.

Moon, Christine, Hugo Lagercrantz \& Patricia K. Kuhl. 2013. Language experienced in utero affects vowel perception after birth: A two-country study. Acta Paediatrica 102(2). 156-160.

Nam, Youngja \& Linda Polka. 2016. The phonetic landscape in infant consonant perception is an uneven terrain. Cognition 155. 57-66. doi:10.1016/j. cognition.2016.06.005.

Narayan, Chandran R., Janet F. Werker \& Patrice S. Beddor. 2010. The interaction between acoustic salience and language experience in developmental speech perception: Evidence from nasal place discrimination. Developmental Science 13(3). 407-420. doi:10.1111/j.1467-7687.2009.00898.x.

Nazzi, Thierry \& Franck Ramus. 2003. Perception and acquisition of linguistic rhythm by infants. Speech Communication 41(1). 233-243. doi:10.1016/S01676393(02)00106-1. 
Nazzi, Thierry, Peter W. \& Elizabeth K. Johnson. 2000. Language discrimination by English-learning 5-month-olds: Effects of rhythm and familiarity. Journal of Memory and Language 43(1). 1-19. doi:10.1006/jmla.2000.2698.

Oh, Janet S., Terry K. Au \& Sun-Ah Jun. 2010. Early childhood language memory in the speech perception of international adoptees. Journal of Child Language 37(5). 1123-1132. doi:10.1017/S0305000909990286.

Patterson, Michelle L. \& Janet F. Werker. 2002. Infants' ability to match dynamic phonetic and gender information in the face and voice. Journal of Experimental Child Psychology 81(1). 93-115. doi:10.1006/jecp.2001.2644.

Patterson, Michelle L. \& Janet F. Werker. 2003. Two-month-old infants match phonetic information in lips and voice. Developmental Science 6(2). 191-196. doi:10.1111/1467-7687.00271.

Partanen, Eino, Teija Kujala, Rosto Nätänen, Auli Liitola, Anke Sambeth \& Minna Huotilainen. 2013. Learning-induced neural plasticity of speech processing before birth. Proceedings of the National Academy of Sciences 110. 15145-15150. doi:10.1073/pnas.1302159110.

Polka, Linda \& Ocke-Schwen Bohn. 1996. A cross-language comparison of vowel perception in English-learning and German-learning infants. Journal of the Acoustical Society of America 100(1). 577-592. doi:10.1121/1.415884.

Polka, Linda \& Ocke-Schwen Bohn. 2003. Asymmetries in vowel perception. Speech Communication 41(1). 221-231. doi:10.1016/S0167-6393(02)00105-X. Polka, Linda \& Ocke-Schwen Bohn. 2011. Natural Referent Vowel (NRV) framework: An emerging view of early phonetic development. Journal of Phonetics 39(4). 467-478. doi:10.1016/j.wocn.2010.08.007.

Polka, Linda, Ocke-Schwen Bohn \& Daniel J. Weiss. 2015. Commentary: Revisiting vocal perception in non-human animals: A review of vowel discrimination, speaker voice recognition, and speaker normalization. Frontiers in Psychology 6. 941. doi:10.3389/fpsyg.2015.00941.

Polka, Linda, Connie Colantonio \& Megha Sundara. 2001. A cross-language comparison of $/ \mathrm{d} /-/ \mathrm{d} /$ perception: evidence for a new developmental pattern. Journal of the Acoustical Society of America 109(5). 2190-2201. doi:10.1121/1.1362689.

Sato, Yutaka, Mahoko Kato \& Reiko Mazuka. 2012. Development of single/ geminate obstruent discrimination by Japanese infants: Early integration of durational and nondurational cues. Developmental Psychology 48(1). 18-34. doi:10.1037/a0025528.

Sato, Yutaka., Yuko Sogabe \& Reiko Mazuka. 2010. Discrimination of phonemic vowel length by Japanese infants. Developmental Psychology 46(1). 106-119. doi:10.1037/a0016718.

Saxton, Matthew. 2000. Negative evidence and negative feedback: Immediate effects on the grammaticality of child speech. First Language 20(60). 221-252. doi:10.1177/014272370002006001. 
Shin, Minha, Youngon Choi \& Reiko Mazuka. 2018. Development of fricative sound perception in Korean infants: The role of language experience and infants' initial sensitivity. PloS one 13(6). e0199045. doi:10.1371/journal. pone. 0199045.

Shultz, Sarah \& Athena Vouloumanos. 2010. Three-month-olds prefer speech to other naturally occurring signals. Language Learning and Development 6. 241257. doi:10.1080/15475440903507830.

Shultz, Sarah, Athena Vouloumanos, Randi H. Bennett \& Kevin Pelphrey. 2014. Neural specialization for speech in the first months of life. Developmental Science 17(5). 766-774. doi:10.1111/desc.12151.

Spelke, Elizabeth S. 1979. Perceiving bimodally specified events in infancy. Developmental Psychology 15(6). 626-636. doi:10.1037/0012-1649.15.6.626.

Streeter, Lynn A. 1976. Language perception of 2-month-old infants shows effects of both innate mechanisms and experience. Nature 259(5538). 39-41. doi:10.1038/259039a0.

Sundara, Megha, Celone Ngon, Katrin Skoruppa, Naomi H. Feldman, Glenda M. Onario, James L. Morgan \& Sharon Peperkamp. 2018. Young infants' discrimination of subtle phonetic contrasts. Cognition 178.57-66. doi:10.1016/j. cognition.2018.05.009.

Tsuji, Sho \& Alejandrina Cristia. 2014. Perceptual attunement in vowels: A meta-analysis. Developmental Psychobiology 56(2). 179-191. doi:10.1002/ dev.21179.

Tsuji, Sho \& Alejandrina Cristia. 2017. Which acoustic and phonological factors shape infants' vowel discrimination? Interspeech 2017, 2108-2112.

Van Lancker, Diana \& Victoria Fromkin. 1973. Hemispheric specialization for pitch and "tone": Evidence from Thai. Journal of Phonetics 1(2). 101-109.

Vikner, Sten. 2001. Det særlige ved sproget er at det er et genetisk determineret organ. Unpublished manuscript. http://www.hum.au.dk/engelsk/engsv/ medfoedt.htm.

Voegtline, Kristin M., Kathleen A. Costigan, Heather A. Pater \& Janet A. DiPietro. 2013. Near-term fetal response to maternal spoken voice. Infant Behavior and Development 36(4). 526-533. doi:10.1016/j.infbeh.2013.05.002.

Vouloumanos, Athena, Marc D. Hauser, Janet F. Werker \& Alia Martin. 2010. The tuning of human neonates' preference for speech. Child Development 81(2). 517-527. doi:10.1111/j.1467-8624.2009.01412.x.

Vouloumanos, Athena \& Janet F. Werker. 2007. Listening to language at birth: Evidence for a bias for speech in neonates. Developmental Science 10(2). 159164. doi:10.1111/j.1467-7687.2007.00549.x.

Watson, Tamara L., Rachel A. Robbins \& Catherine T. Best. 2014. Infant perceptual development for faces and spoken words: An integrated approach. Developmental Psychobiology 56(7). 1454-1481. doi:10.1002/dev.21243. 
Werker, Janet F. 1994. Cross-language speech perception: Development change does not involve loss. In Judith C. Goodman \& Howard C. Nusbaum (eds.), The development of speech perception: The transition from speech sounds to spoken words, 93-120. Cambridge, MA: MIT Press.

Werker, Janet F. \& Richard C. Tees. 2005. Speech perception as a window for understanding plasticity and commitment in language systems of the brain. Developmental Psychobiology 46(3). 233-251. doi:10.1002/dev.20060. 